\title{
ES POSIBLE CONSTRUIR UNA NUEVA PAUTA DE DEBATE PARA LA INVESTIGACIÓN Y EVALUACIÓN DE LAS POLÍTICAS SOCIALES ${ }^{1}$
}

\author{
Carola C. Arregui \\ Pontificia Universidad Católica de São Paulo (PUC-SP)
}

\begin{abstract}
ES POSIBLE CONSTRUIR UNA NUEVA PAUTA DE DEBATE PARA LA INVESTIGACIÓN Y EVALUACIÓN DE LAS
\end{abstract} POLÍTICAS SOCIALES?

Resumen: Este artículo busca situar y problematizar la evaluación introducida en el marco de la Nueva Gerencia Pública (New Public Management). Pretende recuperar los elementos centrales e inherentes a las políticas sociales, abandonados en la perspectiva gerencial y que precisan ser reposicionados para la construcción de un debate sobre los propósitos de la evaluación.

Palabras-clave: Evaluación, políticas sociales, derechos sociales.

IS IT POSSIBLE TO BUILD A NEW AGENDA OF DEBATE FOR THE RESEARCH AND ASSESSMENT OF SOCIAL POLICIES?

Abstract: This paper intends to situate and discuss the assessment introduced on the New Public Management board. The purpose of this work is to recover the core elements inherent to social policies, abandoned in the managerial perspective and that need to be restored for the construction of a debate on the assessment's purposes.

Key words: Evaluation, social policies, social rights 


\section{INTRODUCCIÓN}

Reconstruyendo los hilos tejidos por el mercado. La evaluación como pieza política clave para legitimar las reformas

Durante las últimas décadas del final del siglo pasado el mundo pasó por un proceso vertiginoso y contradictorio de integración/fragmentación y de reconcentración del capital. A partir de las crisis del petróleo de la década de 1970 se inaugura un conturbado período de reestructuración económica y de reajuste social y político sustentados en los principios de apertura-liberalización de las economías, desregulación de los mercados (en particular de los financieros y del trabajo), equilibrio fiscal y estabilidad monetaria, bien como la reducción del papel del Estado.

Desacreditada la fórmula keynesiana, insultada a partir de ahí por estatizante, corporativista y demagógica, el proyecto neoliberal aparece como el gran articulador ideológico y político de un amplio conjunto de reformas de ajuste estructural de enfrentamiento de la crisis. El utilitarismo se transforma en regla de conducta, fomentando la exacerbación del consumo, de los narcisismos, de los inmediatismos y del abandono de los valores de justicia e igualdad que dejan de tener utilidad, en un mundo regido por el cálculo económico y por la competitividad.

Durante la década de 1990, en América Latina la reforma del papel del Estado se expresaría en los conocidos procesos operados de flexibilización, desregulación y privatización de los servicios públicos, bien como en la reducción y contención del déficit fiscal, que, en la práctica, se daría casi que estrictamente en la contención del gasto social. Este proceso significó profundos cambios en lógica de provisión de las políticas sociales, erosionando el sentido de responsabilidad pública del Estado, en países campeones por su desigualdad social.

El redimensionamiento del Estado se daría, igualmente, por medio de los llamados procesos de "modernización" del aparato burocrático y del modo de gestión, condensados en los nuevos principios de organización y estructuración del modelo de la Nueva Gerencia Pública (New Public Management), característico del Estado Neoliberal. Para superar la carga de inoperancia e ineficacia del modelo tradicional de gestión burocrática el nuevo modelo propone introducir "una economía institucional basada en la aplicación de los principios gerenciales del sector empresarial privado en el sector público". De esta forma, las reformas pretendidas buscaban asegurar el desempeño, control y reducción de las cuentas públicas; flexibilizar la organización del Estado para proveer servicios adecuados a los ciudadanos y desarrollar nuevas prácticas, competencias e instrumentos para mejorar la gerencia, optimizadas por las tecnologías de la información. El binomio reducción del gasto público (léase social) y la racionalización del aparato burocrático impondrían las palabras de orden del nuevo modelo: "la gestión por resultados", lo que representará obviamente, como veremos a seguir, en impactos sobre los procesos y la naturaleza de la evaluación que será adoptada en las décadas siguientes.

Omar Guerrero (2004, p. 10) nos alerta sobre la visión privatizadora de lo público subyacente al modelo de la Nueva Gerencia Pública.

Aunque lleva por nombre la voz manejo (management), no es el manejo su fundamento, sino la más general noción de empresa mercantil, y tal es el modelo que propone como sustituto del esquema "burocrático" del Estado. Aquí tienen su origen las propuestas a favor de establecer mercados intra-estatales y orientar al gobierno hacia el consumidor, no hacia el ciudadano, así como la introducción del "espíritu empresarial" en el gobierno y el desarrollo de los principios de la competencia en la provisión de bienes y servicios públicos. Aunque orgullosamente ostenta como apellido el vocablo público, nada lo vincula a esa palabra, pues sus propósitos y resultados se encaminan a la privatización del Estado. Su fruto más palpable constituye una paradoja: el renacimiento de la dicotomía políticaadministración y la emergencia de la antinomia entre policy y management'.

Bajo fundamentos meramente económicos, basados en la empresa privada y la noción del mercado, se procesa la transposición de lo público para lo privado y la pulverización de la ciudadanía en individuos-consumidores que siendo objeto de servicios públicos no son más portadores de derechos, sino de utilidades individuales ahora definidas como satisfacciones. A partir de esta perspectiva, Guerrero analiza la doble dimensión de la privatización como pieza llave para entender los cambios que se procesaron con la constitución de la Nueva Gerencia Pública.

El desmonte de los servicios públicos maximizó la privatización de todo lo que era rentable para el mercado, ampliando los ámbitos de acumulación. De esta forma, el Estado podría quedar concentrado en funciones básicas y esenciales, focalizando los programas sociales en de la población en extrema pobreza y en situación de riesgo social. Paralelamente, se incorporó en la estructura políticaideológica-institucional del aparato del Estado la lógica y los principios del mercado, introduciendo medidas administrativas y prácticas de gestión típicas de la administración de empresas.

De hecho, para Desrosières (2008), la emergencia del Estado Neoliberal derivó en 
profundas transformaciones en la lógica de la gestión pública caracterizada a partir de ese momento por 'la política de los grandes números', calificando el énfasis y la perspectiva del tipo de cuantificación que se tornará dominante tanto del punto de vista de los procedimientos como de la producción de las estadísticas nacionales. De esta forma, ganan espacio en la evaluación de las políticas sociales la producción de datos cuantitativos priorizando los resultados contables, los indicadores de performance y la importancia del establecimiento de la comparación internacional vía producción de rankings. $Y$ del otro, el predominio de modelos econométricos y probabilísticos para la evaluación de las políticas, para la realización de estudios de resultados e impactos de programas pilotos y para la implementación de perfiles de individuos en situación de riesgo.

En este sentido es que la evaluación pasa a ser una pieza llave e central para demostrar que la nueva racionalidad produce buenos resultados, reorganizando y achicando un aparato burocrático inflado, minimizando al máximo los costos y reorientando las políticas sociales para los grupos en situación de riesgo y vulnerabilidad social. Sobre tal perspectiva, se refuerza la identificación entre evaluación y auditorias, la importancia de un riguroso monitoreo por intermedio de sofisticados sistemas de información, la proliferación de indicadores cuantitativos para analizar costos/beneficios y la vinculación del campo de la evaluación con la prestación de cuentas, sobre la influencia progresiva de los economistas y de la perspectiva econométricafinanciera en la producción de los estudios de evaluación de resultados e impactos.

Entretanto, el balance de América Latina, en la entrada del siglo XXI, mostraría la crónica de una concentración anunciada por los diferentes movimientos sociales, sindicales y la izquierda que denunciaban y se oponían a las reformas implementadas. A pesar de las diferencias entre las particularidades históricas, económicas y políticas de cada país, la región completara el ciclo iniciado en las dictaduras cívico-militares: abrió y liberalizó sus economías, desreguló el mercado de trabajo, fomentó un proceso creciente de privatización, promovió la tan deseada estabilidad monetaria y controló firmemente el déficit fiscal. Sin embargo, la aplicación de las reformas no derivó en el prometido crecimiento económico; el Producto Bruto Interno (PBI) anual durante toda la década de los 90 fue $3 \%$, apenas un $1 \%$ a más que en la década perdida de los 80 y bien abajo del $5 \%$ de la década de los 60. Además, no sólo permaneció, como se agravó el cuadro de crisis social expreso en el aumento considerable de las tasas de desempleo, la expansión de la precarización e informalidad del mercado de trabajo, el crecimiento de las tasas de pobreza y la polarización social derivada de la queda del rendimiento de los trabajadores y clases medias y la concentración de la misma en las elites económicas. Las políticas de ajuste neoliberal en América Latina, superpuestas a la herencia social acumulada histórica y estructuralmente, afectaron las políticas sociales en una doble perspectiva: por un lado, se amplió la demanda y se tornó más compleja frente al deterioro de las condiciones de vida de su población, y por otro, se restringió la oferta como resultado de los cortes en el gasto social y de la substitución de la concepción universal por programas focalizados y residuales (ARREGUI, 2005).

En este contexto, gana relevancia en el discurso del nuevo modelo de gerencia del Estado la importancia de mantener la eficacia, la eficiencia y la economía en la gestión, bajo un nuevo desafío: alcanzar la calidad de los servicios. Del punto de vista de la gestión y de la evaluación emergen nuevas estrategias y procedimientos para la construcción de estudios de calidad, servicios al consumidor y satisfacción de los usuarios, separando servicios de derechos sociales y desconsiderando los efectos distributivos y redistributivos de las políticas sociales.

La supuesta modernización de los 90 completa un ciclo de reformas conservadoras, antipopulares, privatistas e con profundo sentido anti-público. La transparencia y la participación ciudadana que los organismos multilaterales predican son juegos discursivos para legitimar la disciplina fiscal y la pulverización de demandas sociales vía la evaluación desarticulada e individual de la calidad, cantidad y oportunidad de beneficios y servicios recibidos.

En este sentido, la evaluación debe ser entendida, no sólo como la introducción de nuevas técnicas de gestión que apoyan la tomada de decisión y la planificación, sino también, como pieza política llave para legitimar las reformas y para promover la incorporación y permanencia del nuevo modelo.

Por eso, es que el Banco Mundial sostiene que los procesos de evaluación y monitoreo

[...] son parte central de los acuerdos para una buena gobernabilidad y necesarios para lograr la formulación de políticas basadas en la evidencia, la toma de decisiones presupuestarias, la administración y la rendición de cuentas. (MAKAY, 2007).

O, que Juan Cristóbal Bonnefoy (2005), jefe del Instituto Interamericano para el Desarrollo del $B I D$, identifique la gestión por resultados no apenas como un problema de medición sino como estrategia para construir "[...] climas sociales favorables hacia la disciplina fiscal [...]", conforme puede ser identificado abajo:

La transición a la gestión por resultados no es sólo un problema de medición sino un cambio de mentalidad. Debemos fortalecer la capacidad del gobierno 
para implementar una política fiscal sostenible y creíble, con el mínimo costo para su eficacia de gestión. Pero también transmitir a los actores los dilemas de la administración pública para generar un clima favorable hacia la disciplina fiscal. (BONNEFOY, 2005).

De esta forma, el campo de la evaluación no puede ser visto nunca como un asunto meramente técnico y/o aséptico. Conforme apuntan varios estudiosos de la evaluación (SILVA, 2008; OFFREDI, 2010), la perspectiva teórica y metodológica que la fundamenta, la formulación de los principios que le dan el norte, bien como los procesos y procedimientos que ella desencadena, están sustentados en una visión de mundo y una dirección que se pretende tomar.

La paradoja en nuestra región es que este proceso se daba en un contexto en donde los sectores populares presionaban para la democratización y desburocratización del Estado y por la defensa de la garantía y ampliación de las políticas y derechos sociales. Esta tensión estaría presente en los procesos de modernización del aparato del estado, que combinó mecanismos de desburocratización, descentralización, participación y control social, al mismo tiempo en que se reforzaba la apropiación privada de lo público.

De otro lado, los cambios en el campo político que se operaron durante la década de los 2000, con la elección de partidos progresistas y/o de tradición de izquierda introducen fuertemente en la perspectiva de las políticas sociales el enfrentamiento de la pobreza y la desigualdad, sin cuestionar, entretanto, el modelo de gestión y evaluación que se ha implementado en nuestra región. Permanece la centralidad de la discusión de la eficiencia y eficacia del gasto, la importancia de los sistemas de información para acompañar las políticas y la proliferación de métodos y metodologías para evaluar los resultados y la calidad de los servicios.

\section{EL PAPEL DE LA INFORMACIÓN EN LA SOCIEDAD DEL ESPECTÁCULO Y DEL RIESGO}

En el contexto de la Nueva Gerencia Pública, la información y la tecnología se tornaron piezas claves para la construcción de una nueva racionalidad. Los objetos tecnológicos, como sabemos, dependen, operan y fornecen informaciones constantemente, tome-se como ejemplo los cambios en la sociedad contemporánea con la introducción masiva de los computadores y celulares.

La cuestión central, como apunta Marilena Chaui (2006), es saber quién detenta la gestión de la masa de informaciones, quién utiliza esas informaciones, cómo y para qué las utiliza. Esta interrogación se torna fundamental para comprender como se está operando la gestión de lo social.
El área social nunca tuvo una tradición en el uso de instrumentos tecnológicos e informacionales en su dinámica y en los procesos de trabajo. Podríamos hablar de no mucho más que de una década para identificar el momento en que los procesos de gestión y evaluación comienzan a exigir de los profesionales nuevos procedimientos y habilidades que les permitan utilizar un conjunto de herramientas como base para las fases de planificación y evaluación de proyectos, programas e políticas sociales.

Si por un lado, como vimos, este impulso resulta, en parte, de la implementación de este nuevo modelo de gestión, también es cierto que los procesos acelerados de urbanización y los cambios que se operaron en las condiciones de vida de la población, exigieron cada vez más un conocimiento mayor para diseñar la intervención a partir de las demandas sociales existentes. El problema central entonces no estaría en el reconocimiento de la necesidad de conocer mejor, sino en responder qué tipo de informaciones son necesarias para la gestión y evaluación de los proyectos y programas y como nos incita Chaui (2006) quiénes las utilizan, cómo y para qué se utilizan.

Retomemos entonces el contexto de reformas estructurales que se operaron en América Latina, a partir de las décadas de los 1990 y el discurso de mejor locación del gasto social en los sectores más desfavorecidos. Aquí radica el argumento y la práctica de focalización de programas y servicios sociales en los sectores ya denominados, en la época, por los organismos multilaterales, como grupos en situación de riesgo social. En este contexto las medidas de pobreza fueron especialmente utilizadas para la localización de los recursos y programas en los más pobres entre los pobres, creando, como problematiza Sposati ([2000]), una nivelación siempre para abajo como referencia de la pobreza².

Nacen así un conjunto de medidas e indicadores sociales que buscan de un lado, caracterizar y clasificar el "público-alvo" de los programas sociales y del otro, analizar los impactos de las acciones desencadenadas.

Entretanto, la proliferación a lo largo del tiempo, y, principalmente, en los últimos años, de diversas propuestas metodológicas y su uso cada vez más frecuente en la gestión de las políticas públicas, no ha derivado, necesariamente en cambios sustanciales con relación a las profundas desigualdades sociales. Parafraseando Rosanvallon (2011, p. 19), "[...] sabemos cada vez más, hablamos cada vez más sobre la desigualdad, pero nada cambiamos."

¿Cómo explicar esta paradoja? Hasta qué punto está clara la relación entre las medidas y las metodologías producidas, las concepciones de sociedad y las formas de la acción pública sobre la realidad? Si, de un lado, se ha avanzado en la crítica 
densa sobre los límites de la perspectiva monetarista en la construcción de las medidas de desigualdad y pobreza, es posible afirmar lo mismo, con relación a la cacofonía de metodologías no monetaristas surgidas en los últimos 20 años?

Hasta la década de 1990, la perspectiva monetarista fue dominante en la construcción de las medidas de pobreza y desigualdad, operando un conjunto diverso de proposiciones, algunas vigentes hasta el día de hoy, de cuantificación de la pobreza a partir de las dimensiones de la renta y/o del consumo, estableciendo padrones mínimos de vida traducidos en un valor monetario. En décadas posteriores, cuestionando la visión restricta y unidimensional de la perspectiva monetarista, surgieron proposiciones que buscaron estructurar cuadros analíticos con énfasis en el carácter multidimensional de la pobreza, destacando otros aspectos como el acceso o la falta de acceso a servicios sociales, a espacios de participación, poder y a la posibilidad de acceder de la riqueza material e inmaterial socialmente construida.

De alguna forma, a lo largo de las últimas décadas, vía las diversas experiencias y proposiciones, se fue construyendo un consenso sobre el carácter incompleto de la dimensión de la renta para el análisis de los fenómenos relativos a la desigualdad y a la pobreza. Por otro lado, la perspectiva monetarista es todavía referencia en América Latina como parámetro, por ejemplo, para la implantación de los programas de erradicación de la pobreza, y especialmente para los programas de transferencia de renta condicionada.

Una investigación en curso sobre los programas de transferencia de renta condicionada en América Latina y el Caribe, realizada por universidades brasileñas, argentinas y uruguayas ${ }^{3}$ llama la atención para la complejidad y sofisticación de los procedimientos existentes en gran parte de los países para la selección, acompañamiento y control de las condicionalidades de la familias atendidas. Alteraciones tecnológicas e institucionales que impactan tanto el campo de la gestión de lo social como los procesos de trabajo profesionales, destacando que la tecnificación de los procesos de acompañamiento y evaluación de las familias beneficiarias de los programas han implicado, no sólo costos elevados para construir, implementar y alimentar los sofisticados sistemas de información, como también un control sistemático de las familias, que prioriza lo cuantitativo en detrimento de lo cualitativo. Así hay una suerte de sobredimensionamiento de la tecnología y los procedimientos matemáticos y estadísticos como factores aparentes de una racionalidad que dispensa la mirada, el análisis y la cualificación de de la intervención profesional.

En estudios anteriores destacábamos que uno de los rasgos que ha caracterizado la gestión de lo social en América Latina desde la década de los 90 es la introducción del concepto de vulnerabilidad y su asociación con la dimensión del riesgo. Tal es así y permanece vigente hasta el día de hoy que el estudio comparado revela que las categorías centrales para la elección del "público-alvo" de los programas de transferencia de renta son la pobreza, la extrema pobreza y la vulnerabilidad.

Interesa a los efectos de esta discusión retomar algunas consideraciones ya realizadas (ARREGUI; BELFIORE WANDERLEY, 2009) sobre los posibles impactos de la asociación de la pobreza con la dimensión del riesgo. El saldo social del modelo neoliberal implementado en América Latina creó problemas para los think tanks de los organismos internacionales que tuvieron que asumir y explicar el fracaso de los planes de reforma para la región; sin cuestionar el modelo, reconocían el agravamiento de las condiciones sociales de nuestra región. Hacia final de la década de 90 se tejió una nueva unanimidad: la vulnerabilidad social se tornó la palabra-clave para explicar la pobreza en América Latina. La vulnerabilidad aparece, así, como un concepto que trasciende la visión miope de la pobreza y se torna base y estrategia para la construcción de propuestas metodológicas de comprensión, medición y diseño de programas de combate a la pobreza.

Mismo que el discurso sea el de agregar nuevas matices aparentemente inexistentes en concepto de pobreza, o inclusive el de transcender la lectura de las carencias, el nuevo concepto no consigue desembarazarse de los aspectos negativos, que tradicionalmente se asocian a la pobreza. Así, la vulnerabilidad es entendida o como desequilibrio entre activos de familias e individuos y la estructura de oportunidades de una sociedad (KAZTMAN; FILGUEIRA, 1998/1999), o como área de riesgo que se sitúa entre la integración plena y la exclusión total (BUSSO, 2001) o mismo como debilidad, desventaja y problemas para el desempeño e movilidad social de los individuos (COMISIÓN ECONÓMICA PARA AMÉRICA LATINA Y EL CARIBE, 2001).

De hecho, el análisis de la bibliografía permitiría abrir un abanico infinito de definiciones, sin producir, entre tanto, un marco teórico conceptual claro en torno de este concepto. Pero es posible identificar un común denominador entre un conjunto variado de definiciones: la asociación del concepto de vulnerabilidad con el de riesgo social.

Ya anotábamos en aquella oportunidad que esto no es una perspectiva nueva. Históricamente la pobreza ha sido considerada como caso de policía, y, por tanto, asociada con clases peligrosas y foco de estrategias represivas o da vigilancia social. De hecho, esto representa hasta la actualidad el tratamiento clásico de la cuestión social revelando un sistema que no es capaz de resolver su principal contradicción: mientras que la producción social y el trabajo son colectivos, la apropiación de sus frutos queda monopolizada en una parte da sociedad (IAMAMOTO, 1998). O en las palabras de Otávio lanni (1991) el mismo sistema que genera progreso y riqueza para algunos, genera exploración y pobreza para otros. 
El avanzo en la legislación e intervención de políticas sociales que abordan la pobreza como expresión de las desigualdades económicas, sociales y culturales convive, hasta los días actuales, con matrices conservadoras. A pesar de que avanzamos en materia de conquistas de derechos sociales, continúa siendo moneda corriente la tutela sobre los pobres (como la exigencia de las condicionalidades en los programas de transferencia de renta), la estructuración de estrategias higienistas sobre el ropaje de programas sociales (especialmente cuando se trata de población en situación de calle) o el simple exterminio social (cuando vemos la notoria diferencia del índice de muertos por homicidios violentos entre las periferias de las grandes ciudades e de los barrios nobles). (IANNI, 1991, p. 4).

El primer problema de esta nueva reedición $y$ abordaje es identificar la vulnerabilidad social con pobreza sin tejer las relaciones necesarias con la cuestión de las desigualdades y de la distribución de la riqueza. Incorporar esta noción acríticamente supone, sí, un riesgo: el de quedar atrapados en una intervención mecanicista y clasificatoria, haciendo de cuenta que no existen cuestiones estructurales que condicionan la cuestión social. El segundo, es que al descontextualizar la discusión de la pobreza y de la desigualdad se refuerza la tendencia de individualización de las cuestiones sociales y la desresponsabilización de la cosa pública.

Asociar la pobreza con desventaja, debilidad $y$, principalmente, con riesgo, en una sociedad que asocia riesgo con miedo, incerteza e inseguridad, puede derivar en la retomada de estigmas que asociaban y asocian pobres con clases peligrosas, $y$, por tanto, reforzar intervenciones represivas $y$ tutelares.

Mismo con el mundo mediático promoviendo lo contrario, puesto que usa y abusa de la asociación de la noción del riesgo como amenaza y peligro, generalmente asociada al espectáculo de la violencia urbana y de los sectores populares, parte de la intelligentsia académica ha conseguido construir una pretensa aura de neutralidad e asepsia en torno del concepto de riesgo, como si este fuese un elemento constitutivo de los individuos o de las cosas, y no una construcción social y cultural.

Para Castel (2005, p. 59), el concepto de peligrosidad fue substituido por el de riesgo, y, con esto, cambiaron las estrategias contemporáneas de gestión de lo social. Estrategias que disuelven la noción de sujeto colectivo concreto y ponen en su lugar una combinación de factores, los factores de riesgo. 'Un riesgo no surge de la presencia de un peligro específico personificado en un grupo o individuo concreto. Es el efecto de una combinación de factores concretos que produce la mayor o menor probabilidad de ocurrencia de modos de comportamiento indeseables. Ser designado 'en situación de riesgo' es ser localizado en el medio de una red de factores extraídos de experimentos y modelos matemáticos. Del punto de vista de la gestión Castel sostiene que esto está produciendo un pasaje de la tradicional intervención social que suponía proximidad, una relación cara a cara para políticas genéricas de gerenciamiento de poblaciones. De ahí, la importancia de edificar un modo de vigilancia basado en el monitoreo de datos estadísticos de los territorios, des-construyendo el sujeto de intervención e reconstruyéndolo vía combinación de factores plausibles de producción de riesgos.

Y este es el cuadro que precisa ser evocado cuando se debaten las concepciones que dominan las medidas y los instrumentos de evaluación y monitoreo que vienen surgiendo en los últimos años. Como Carla Bronzo (2005) afirma, detrás de una propuesta de medición, hay una concepción que lanza luz para algunos aspectos de la realidad, pero también, deja en la sombra otros. Y agrega, que las concepciones producen diferentes formas de medición, pero también e igualmente diferentes respuestas en relación a la formulación e implementación de las políticas sociales.

\section{LA NECESARIA RECONSTITUCIÓN DE LOS PROPÓSITOS DE LAEVALUACIÓN: ¿es posible} la independencia del debate latinoamericano?

Más allá de la clara y evidente importancia del uso de informaciones en el proceso de planificación, ejecución y evaluación de las políticas sociales, surge en la actualidad un conjunto de cuestionamientos sobre el tipo y la cantidad de datos que están siendo producidos.

Paulo Januzzi (2011) hace referencia a la paradoja de la "escasez en la abundancia" para denominar el proceso creciente, y muchas veces desarticulado, de la producción de informaciones en el ámbito de la gestión. Son cada vez más los datos colectados en los diversos sectores, departamentos de las diversas políticas sectoriales o en los diferentes ámbitos de la gestión pública $y$, sin embargo, continuamos afirmando que no tenemos la información suficiente o relevante para apoyar los procesos de decisión y diseño de los diversos programas y proyectos sociales. De esta forma, la producción creciente de datos cuantitativos no ha permitido que el mayor conocimiento de la realidad social resulte en cambios substanciales en las políticas sociales.

A pesar de haber aumentado la producción y densidad de los bancos de datos y de haber construido sofisticados sistemas de información, sean para analizar las condiciones de vida de la población o para evaluar el desempeño de las 
políticas sociales, los resultados no dan cuenta de la complejidad de la realidad, ni de los cambios que se están procesando. El problema central para Januzzi (2011) es recuperar la capacidad de medir lo que es relevante, significativo y útil, creando insumos esenciales para el conocimiento de la realidad y para la gestión pública. Es tal el dilema que colectamos y producimos cada vez más información, pero escuchamos cada vez menos a la población destinataria de los programas.

En este sentido, existe en San Pablo, un grupo de estudiosos y profesores, que también han participado en el campo de la gestión, que realizan una fuerte crítica a la lógica de la producción de las estadísticas e indicadores sociales puesto que al desconsiderar aspectos vitales de las condiciones de vida de la sociedad y sus dinámicas, no revelan ni traducen la complejidad de las cuestiones sociales emergentes y las especificidades y disparidades que se diseñan en los contextos poblacionales y territoriales (SPOSATI, 2001; KOGA, 2003). La tendencia de homogeneización inherente al proceso de cuantificación resulta en la homogeneización de la realidad, contrastando con el carácter multidimensional y multifacético de las cuestiones sociales.

Indican, así, la necesidad de agregar al lente estadístico el lente de la dinámica social. Para tanto, realizan estudios y elaboran proposiciones de medidas que buscan entender las desigualdades clásicas y estructurales, en el contexto de las nuevas configuraciones de precarización de la población y de los territorios urbanos. En este sentido, construyeron el Mapa de Exclusión/Inclusión de la ciudad de San Pablo, por medio de medidas intra-urbanas capaces de revelar las desigualdades y las discrepancias sociales y con el objetivo de incidir en el horizonte de inclusión social. La dimensión territorial no es una referencia de localización apenas, sino texto y contexto en donde se producen y reproducen las relaciones sociales. De ahí que incorporen la noción de Topografía Social para identificar y comprender las relaciones, entrabes y tensiones entre las demandas detectadas y la real oferta de políticas sociales (SPOSATI, 1996) 4 .

De hecho, la sociedad y las ciudades están pasando por profundas transformaciones que precisan ser comprendidas. Pero como afirman, Vera Telles y Roberto Cabanes (2006, p. 78), esto implica un doble desafío pues

[...] la construcción de parámetros críticos implica también la construcción de parámetros descriptivos para colocar en perspectiva las realidades urbanas en mutación.

De hecho los procesos de construcción de datos en el ámbito de la gestión y evaluación han priorizado las dos puntas de la cadena de informaciones (en un extremo bases de datos sobre las condiciones de vida de la población y en otro extremo sobre resultados e impactos de las políticas), creando un vacío en la dimensión analítica del seguimiento de las políticas sociales (JANUZZI, 2011). Cómo superar estos entrabes, en un contexto que presiona permanentemente por la producción incesante de nuevos y novedosos indicadores cuantitativos.

Dialogamos, para tanto, con la experiencia europea. En el debate francés, el exceso de información cuantitativa deriva en lo que Vincent de Gauléjac (2005) denomina como cuantifrenia, para criticar el uso exacerbado de la cuantificación y problematizar el predominio de una lógica instrumental, que exige la producción incesante de las informaciones, olvidando la necesaria interrogación anterior que permitiría identificar para qué sirven y a qué se destinan los datos creados. La supremacía de indicadores cuantitativos en la perspectiva financiera y económica desconsidera las competencias específicas y el sentido público de las políticas sociales.

Contraponiéndose a esta perspectiva, en 2009, surgió en Francia un movimiento social integrado inicialmente por trabajadores de la salud y luego extendido a otras áreas de políticas sociales, militantes, universitarios e investigadores, que cuestionan la lógica subyacente al modelo de la Nueva Gerencia Pública, las reformas y cortes presupuestales implementados en los diversos servicios y los métodos utilizados para la evaluación.

El movimiento l'Appel des appels5 critica la concepción privatista, gerencial y tecnocrática del modelo dominante de gestión y de evaluación que fragmenta e instrumentaliza no sólo a los sujetos colectivos y destinatarios de las políticas sociales, como también a los agentes y a las prácticas de los profesionales que las operan. Las nuevas formas de evaluación moldan y recomponen todos los asuntos relativos al bien común, al cuidado de la salud, a la educación, a la justicia, a la cultura, etc. Es por medio de un dispositivo instrumentalizado, racionalizado y técnico que se exige a los profesionales una nueva forma de pensar sus prácticas, obligándolos a considerar los servicios como productos financieros, no haciendo otra cosa que evaluar medios, para poder ignorar sentidos e finalidades. Es por este dispositivo que se confunde valor con clasificación, que se substituye el raciocinio por el manual y que se confisca vía procedimientos, el pensamiento y la decisión del profesional que opera la política social.

En el mismo año, surge otro movimiento completamente diferente del anterior, que, cuestionando la lógica de los indicadores macroeconómicos (como el Producto Bruto Interno $(P \mid B))$, reivindica, sobre el nombre de «nuevos indicadores de riqueza», la necesidad de construir otro tipo de estadísticas para pensar la noción 
de crecimiento, contemplando aspectos vitales que midan el real bienestar de la sociedad, como los efectos de las desigualdades sociales y la degradación ambiental y climática. Se configura así, el Fórum pour d'autres indicateurs de richesse (FAIR)6, integrado por militantes de asociaciones y sindicatos, actores locales e investigadores de diversas disciplinas, que proponen una doble perspectiva de actuación. Una de orden conceptual y ética, puesto que, sobre la consigna 'más allá del PBI', enfatizan la necesidad de ultrapasar los valores que están en la base de los indicadores monetarios que dan prioridad solamente al valor de la producción del mercado y la otra de orden político ya que el proceso de elaboración de esta nueva construcción presupone abrir el debate público para definir cuáles son los fines que deberán ser considerados.

Como alerta Patrick Viveret (2009), no se trata solamente de una simple discusión técnica, sino de una articulación para promover un amplio debate sobre las decisiones necesarias que marcarán los rumbos de la sociedad. Esto es especialmente relevante en el contexto europeo que al ser uno de los epicentros de la explosión de las crisis económicas internacionales a partir de 2008 resiste al agravamiento de las cuestiones sociales, con índices inéditos de desempleo y a la presión de las fórmulas neoliberales de desmonte de los estados de Bienestar Social.

Por su vez, en América Latina, la inédita reducción de la pobreza y desigualdad, en un contexto mundial de su recrudecimiento, ha derivado en el mito sobre el crecimiento de las clases medias y sobre la capacidad de consumo de su población ${ }^{7}$. En el contexto brasileño el análisis de la evolución del mercado de trabajo, realizado por Mario Pochmann (2012) muestra que, a diferencia de la década anterior en la década de los 2000, creció el empleo y creció el empleo formal. Entre tanto, cuando analiza ese crecimiento a partir de las franjas de renta, apunta que la mayor parte se dio en empleos donde los trabajadores no alcanzan a ganar dos salarios mínimos, con lo cual problematiza el mito de la clase media. Así, no son pocos los cuestionamientos sobre el destino pretendido para el desarrollo económico y social de una América Latina que redujo su extrema pobreza (del punto de vista de los indicadores monetarios), que adquirió nuevas capacidades de consumo, pero en donde permanecen las fracturas y desigualdades estructurales, las inmensas disparidades de renta, la brutal segmentación de los mercados de trabajo y la destitución de derechos básicos de ciudadanía.

Estos debates no sólo obligan a repensar las lógicas del modo de producción y organización del sistema capitalista, como también colocan en la pauta la necesidad de pensar cuáles deben ser las prioridades en la gestión y evaluación de las políticas sociales.
Coincidimos con Boschetti (2009), que si discutimos la evaluación apenas como un enmarañado cada vez más sofisticado de métodos y técnicas evaluativas, desconsideramos las relaciones y mediaciones que se procesan entre mercado, Estado y políticas sociales y sus funciones y papel en la producción y reproducción de las desigualdades sociales.

De hecho, la perspectiva funcionalista, economicista y gerencial de la Nueva Gerencia Pública esconde cuestiones centrales del campo de la evaluación de las políticas sociales. El énfasis evaluativo de las últimas décadas, que priorizó el estudio de la eficacia, de la eficiencia y de los impactos producidos, sobre la perspectiva de mercado de costo/beneficio, abandona el principio de justicia social, inherente a las políticas sociales, desconsiderando así los criterios de igualdad, universalidad y gratuidad.

Además, los criterios para la evaluación de las políticas públicas no pueden tener la misma intencionalidad que los del mercado, de reducción de costos a cualquier precio. Como afirma Boschetti (2009, pag.08), es justamente lo contrario, puesto que si estamos hablando de políticas sociales, que tienen por base la concretización de derechos sociales, la evaluación

[...] debe ser orientada por la intencionalidad de apuntar en qué medida las políticas y los programas sociales son capaces y están consiguiendo expandir derechos, reducir desigualdades y propiciar equidad.

Esto nos lleva a otro elemento central de la discusión. Diferentemente de los que nos proponen los manuales, Evaldo Vieira (2004) alerta para la dimensión de totalidad entre política social y política económica, mismo que formalmente se diferencian o nos den la engañosa impresión de que son cosas diferentes. $Y$ agrega que no se puede evaluar la política social sin remeterse al desenvolvimiento económico, ni se puede analizar la política económica sin detenerse en la política social (VIEIRA, 2004).

En ese sentido, es fundamental apuntar la artificialidad y la engañosa separación que en ámbito de los manuales de evaluación se hace entre análisis de política y evaluación de política, como si la evaluación de la política no demandase el análisis de la política. Y todavía se sugiere una suerte de fractura entre los tiempos de gestión y tiempos de la investigación evaluativa, como si el tiempo de la investigación fuera una pérdida de tiempo, en una sociedad donde el tiempo vale oro. Evaluación e investigación deben permitir acompañar y readaptar programas y políticas pero también responder a cuestiones pertinentes sobre la capacidad de distribución y redistribución de las políticas sociales. 
Sobre tal perspectiva, es de fundamental importancia recuperar la dimensión política que ha venido siendo escamoteada por el proyecto neoliberal y que derivó en la tecnificación de las políticas sociales y despolitización de las prácticas sociales. Romper con la lógica mecanicista representa re-signficar la dimensión pública de la política social.

Recuperar la dimensión política y de totalidad, dislocando el campo de la evaluación del control nominal y numérico de la oferta o vinculada estrictamente a los resultados, permitirá firmar la evaluación como exigencia democrática, contribuyendo para la producción del debate público sobre el sentido, relevancia y pertinencia del enfrentamiento de las cuestiones sociales (OFFREDI, 2010) ${ }^{8}$.

Conforme sostienen Anne Le Roy e Claudine Offredi (2011), estamos frente a un momento donde es necesario revisitar y redefinir tanto los contenidos, como los métodos. La exigencia de la renovación en la evaluación creció junto con la evolución de nuevas demandas, de nuevos sistemas de valores que no los mercadológicos y de la necesidad de una nueva jerarquía entre los elementos que la fundamentan.

En la experiencia francesa el componente socio-político y la legitimidad de los actores que participan del debate han sido construidos progresiva y estratégicamente. La construcción de nuevas perspectivas no admite ser asunto apenas de un grupo selecto de economistas, puesto que es necesario una perspectiva pluri-disciplinaria para cambiar la perspectiva construida hasta el momento; ni siquiera un trabajo de especialistas o de funcionarios del gobierno, puesto que es imprescindible interrogar y construir socialmente lo que es importante de ser evaluado/medido y las finalidades de la vida en sociedad. De esta forma, sostienen la necesidad de calificar, antes de cuantificar. Integrar a la sociedad y a los sectores sociales involucrados es condición para la apropiación colectiva y para la construcción de la legitimidad junto a los ámbitos de decisión.

Trata-se de construir socialmente una mirada sobre la sociedad, donde diferentes actores participen de la construcción del debate público. Para Gadrey (2011) es la forma de recuperar la visibilidad y las fuerzas en tensión de una realidad social que ha sido sistemáticamente dejada de lado. Y al mismo tiempo, la estrategia para construir una perspectiva, que quiebre con la lógica del ciudadano de segunda clase, analizado apenas como patrimonio negativo, como si no existiesen capacidades de expresión y creación social, económicas, políticas y culturales, ni vínculos de solidaridad, resistencia y de lucha, que deben formar parte de los procesos de evaluación y de la construcción de las medidas.

Lo que se asocia con un proceso necesario de análisis, por el cual las relaciones de desigualdad y pobreza deben ser entendidas y discutidas de forma indisociable con la producción de la riqueza.
Landais (2007) e Gadrey (2006) argumentan que en el actual contexto de aumento de las desigualdades en Europa y de la explosión de las altas rentas en el mundo, es necesario repensar los indicadores que miden las disparidades de la renta. Las metodologías tradicionales que comparan la desigualdad a partir de la renta de los $10 \%$ más ricos y de los $10 \%$ más pobres, no son más suficientes para capturar las reales distancias entre los niveles de renta. Ambos coinciden en que es necesario ampliar el lente para capturar las rentas de los muy, muy ricos, porque son ellos los que se están beneficiando de los procesos implementados en las últimas décadas. En un estudio sobre las altas rentas en Francia, Landais muestra que entre 1998 a 2006, mientras que para el $90 \%$ de los hogares de los menos ricos la renta había crecido en torno de $5 \%$, para los $10 \%$ más ricos había aumentado $11 \%$. Sólo que al ampliar las fajas, quedó en evidencia que para $0,01 \%$ de los más ricos la renta había aumentado $43 \%$ ! Fue justamente esta desagregación, dice Landais (2007, p. 5), que permitió capturar el inmenso hiato entre rentas y ayudó a identificar la explosión de las altas rentas de los muy ricos en el país.

Traer para el debate estos elementos permite la construcción de las convenciones sociales como proceso por intermedio de los cuales se fijan los rumbos de la medición, que para Desrosiéres supone explicitar: quien participa, a partir de que nociones e para qué medir?

Como resultado de una convención social la perspectiva de la evaluación no puede desconsiderar el estudio de los usos sociales y de las retóricas de interpretación de la realidad y supone tanto una dimensión técnica como social y política puesto que

[...] las medidas no fornecen solamente un reflejo del mundo (punto de vista más usual) sino que crean una nueva forma de pensar, de representar, de expresar el mundo y de actuar sobre él. (DESROSIÈRES, 2008, p. 13).

Este proceso social y político es lo que para Telles (2004) representa el carácter normativo de la construcción de las medidas sociales, pues lejos de ser una cuestión amparada apenas en criterios científicos, envuelve necesariamente disputas, en el campo práctico y político de la acción y de la intervención pública, para la definición de los valores, concepciones y convenciones que las fundan. Este campo de referencias se torna parámetro para conocer, describir, medir e evaluar la vida social y sus descompases. $Y$ es también el juego de referencias de la crítica social por intermedio de la cual se comprende y problematiza la realidad.

Sin el prisma o la medida por la cual el mundo social gana zonas de relevancia, pertinencia, importancia, el pensamiento termina por vaciarse de su potencia 
crítica para atenerse apenas a la constatación de hechos, cosas, índices, informaciones que se pueden acumular infinitamente, sin llegar al discernimiento de las líneas de fuerza entramadas en el tejido social. (TELLES, 2004, p. 2).

Recuperar el sentido público del campo de la evaluación representa construir una perspectiva al servicio de la democracia, capaz de denunciar las disparidades, los privilegios y criticar las políticas injustas.

Como afirman Anne le Roy y Claudine Offredi (2011, p. 197) el desafío está en la construcción de un nuevo conocimiento de referencia para las políticas públicas y para la sociedad, en donde el cambio de la perspectiva y la posibilidad de promover dinámicas diferentes presuponen la construcción de un sistema pluralista de miradas sobre la realidad que movilice el mundo académico, político y social. El desafío democrático reposa en la capacidad de construir un acuerdo entre las diferentes miradas sobre lo que cuenta verdaderamente en la discusión, y ésta es la condición de legitimidad del proceso.

\section{CONCLUSION}

Como vimos, la institucionalización de sistemas de monitoreo y evaluación en América Latina se relacionan con los grandes procesos de reformas desarrollados en la última mitad del siglo XX. En la década de los 90 , bajo los lemas de eficiencia del gasto público, reducción de la pobreza y transparencia en la prestación de cuentas como vectores estratégicos para el aumento de la legitimidad y de la buena gobernanza, se inauguraron las primeras experiencias de implantación de sistemas nacionales en diversos países de la región. En los años subsecuentes florecieron experiencias similares en gran parte del resto de América Latina. A pesar de la diversidad de herramientas y sistemas existentes, del grado de institucionalización alcanzado y de las grandes diferencias económicas, políticas, sociales y culturales de los países de la región, el Banco Mundial ${ }^{9}$ (GRAU; BOZZI, 2008) al mejor estilo del Consenso de Washington continúa promoviendo la receta única: el impulso y fortalecimiento de la disciplina fiscal y su estrecha relación con una gestión y evaluación en función de los resultados.

Es innegable el crecimiento del campo de la evaluación de las últimas décadas. Al ganar institucionalidad en el área de la gestión pública el campo de la evaluación tiene la oportunidad de ganar relevancia como proceso racional y objetivo que apoya las funciones de la planificación, destacando, como afirma Silva (2008, p. 160) su doble dimensión: técnica y política. De esta forma representa además de

[...] mecanismos para la elaboración y redimensionamiento de programas y políticas, una estrategia de construcción de ciudadanía, de la esfera pública y de la creciente democratización de la sociedad.

También es cierto que si de un lado ha crecido $y$ ha ganado institucionalidad, de otro emergen polémicas y debates sobre la evolución de la evaluación en el actual contexto de crisis internacional y el sentido y dirección que ha tomado en las últimas décadas.

El profesor alemán Hans-Ulrich Derlien (2001), analizando el panorama internacional, afirma que en el campo de la evaluación es posible detectar un proceso contradictorio de expansión, pero al mismo tiempo, de retroceso e, inclusive, de declive que se agudiza en los momentos de crisis económica internacional.

Esto porque el proceso de expansión creciente y su incorporación en la gestión pública se dio afirmándola como pieza clave para la legitimación de las reformas de estado y la reasignación del gasto social, desconectando la política de las políticas sociales. Al mismo tiempo, en los momentos de crisis se reconfiguran las circunstancias distributivas, se reeditan los cuestionamientos cuanto a la validez y la utilización de las evaluaciones y se redefinen las nuevas intencionalidades evaluativas y sus procesos, bien como los actores considerados como legítimos para su implementación. En este contexto, Derlien (2001) sitúa el pasaje del énfasis de la evaluación de impacto (que se reveló poco efectiva y muy costosa) para la evaluación de la oferta y consecuentemente la preocupación por las cuestiones de calidad. Paralelamente, anota cambios en la intensidad y el énfasis de la evaluación producida en la propia estructura pública estatal, inclusive en países como los EEUU, gran impulsor del campo de la evaluación, dando pasaje para un protagonismo mayor de las empresas y consultores privados.

En el caso de América Latina, el estudio del Banco Mundial (GRAU; BOZZI, 2008, p. 18) revelaba, en 2008, que a pesar del crecimiento de la evaluación en todos los países, algunos poseían tan sólo sistemas de monitoreo únicamente, sin haber fortalecido la dimensión de la evaluación, en otros casos mismo bajo la denominación de sistemas existían en la realidad un conjunto de herramientas o de monitoreo y/o de evaluación, sin representar necesariamente un sistema; y el grado de institucionalización de la evaluación variaba enormemente, inclusive porque no había en términos nacionales ni mucho menos como región una definición clara de lo que fuese y representase el papel de evaluación en las políticas sociales.

Propiciar un debate sobre la centralidad política de los derechos sociales en la perspectiva de la evaluación, nos permitiría recuperar la potencia crítica y simbólica de la justicia y la igualdad, como referencias por intermedio de las cuales las demandas y disputas ganarían visibilidad y legitimidad en el palco público (TELLES, 2004). 
Las nociones de justicia e igualdad, según Rosanvallon (2011), presuponen cualidades democráticas y no apenas referencias para las medidas de distribución de riquezas. El desafío es resignificarlas en un contexto que coloca en el centro de la escena ya no más al sujeto de derechos, mas al individuo, por no decir consumidor, en una sociedad que afirma apenas protocolarmente la justicia; que confunde derechos y política con caridad y filantropía; y donde el análisis y descripción da realidad social se presenta como hiperfragmentada y desconectada. El mayor dilema tal vez, como afirma Telles (2004), es reactivar el sentido político y crítico de los derechos y de la igualdad, de modo que las diferencias y asimetrías puedan ser formuladas como cuestiones pertinentes al destino de la sociedad.

Promover este debate, por lo tanto, no está apenas referido a cambios necesarios en la perspectiva de la gestión y la evaluación, mas sobre todo, a una indagación profunda sobre el actual modo de producción y de la propia noción de desarrollo. Conforme afirma Viveret (2009, p. 5), la actual crisis mundial es apenas la punta de un gigantesco iceberg que emerge y revela la experiencia de una gran fractura histórica y el fin de un ciclo que se está produciendo. Para este autor las cuestiones vitales serán aquellas que permitirán responder sobre cómo salir de este gran período histórico, llevando junto lo mejor de la modernidad (la emancipación, la libertad de la consciencia y la duda metodológica) y dejando para atrás lo que tuvo de peor, como a cosificación sobre todas sus formas, siendo la mercantilización y la exploración una de sus grandes características.

\section{REFERÊNCIAS}

ARREGUI, Carola C. O difícil exercício da renda mínima no Brasil. Tese (Doutorado en Servicio Social) - Programa de Pós-Graduacão em Serviço Social, Pontificia Universidade Católica de São Paulo, São Paulo, ago. 2005.

; BELFIORE WANDERLEY, M. A vulnerabilidade social é atributo da pobreza? In: Revista Serviço Social \& Sociedade, São Paulo, ano 30, n. 97, p. 143-165, 2009.

BONNEFOY, Juan Cristóbal. La gestión por resultados: una perspectiva de gerencia pública. Guatemala, 2005. Documento em Power-point. I Curso-Seminario Políticas presupuestarias y gestión por resultados.

BOSCHETTI, I. S. Avaliação de políticas, programas e projetos sociais. In: CONSELHO FEDERAL DE SERVIÇO SOCIAL/ASSOCIAÇÃO BRASILEIRA DE ENSINO E PESQUISA EM SERVIÇO SOCIAL.
Serviço Social: direitos sociais e competências profissionais. Brasilia, DF, 2009.

BRONZO, Carla L. C. Programas de proteção social e superação da pobreza: concepções e estratégias de intervenção. 2005. Tese (Doutorado em Sociologia e Política) - Universidad Federal de Minas Gerais, Facultad de Filosofía y Ciencias Humanas, Belo Horizonte, 2005.

BUSSO, Gustavo. La vulnerabilidad social y las políticas sociales a inicio del sigloXXI. In: SEMINARIO INTERNACIONAL SOBRE LAS DIFERENTES EXPRESIONES DE LA VULNERABILIDAD SOCIAL EN AMÉRICA LATINA Y EL CARIBE, 2001, Santiago de Chile. Anais... Santiago de Chile: CEPAL, 2001.

CASTEL, Robert. A insegurança social: o que é ser protegido? Petrópolis, RJ: Vozes, 2005.

CHAUI, Marilena. Simulacro e poder: uma análise da mídia. São Paulo: Fundação Perseu Abramo, 2006.

COMISIÓN ECONÓMICA PARA AMÉRICA LATINA Y EL CARIBE. Centro Latino-Americano E Caribenho De Demografia. Vulnerbilidad sociodemográfica: viejos y nuevos riscos. Santiago de Chile, 2001.

DERLIEN, Hans-Ulrich. Una comparación internacional en la evaluación de las políticas públicas. Revista do Serviço Público, Brasília, DF, ano 52, n. 1, p. 105-124, jan./mar. 2001.

DESROSIĖRES, Alain. L'argument statistique II : gouverner par les nombres. Paris: Presses de L'école des Mines, 2008.

GADREY, Jean. La richesse et le patrimoine des «exclus». In : MUSEES: exclusion et solidarités, 2011, Paris. Anais eletrônicos... Paris : Fédération des Écomusées et Musées de Société, novembre 2011. Disponible en:<http://www.idies.org/index. php?category/FAIR>. Acceso en: 10 abr. 2012.

Les conventions de richesse au coeur de la comptabilité nationale. In: EYMARDDUVERNAY, F. (Dir.). L'économie des conventions: méthodes et résultats. Paris: La Découverte, 2006

GAULÉJAC, Vincent de. La société malade de la gestion. Paris: Éditions du Seuil, 2005.

GRAU, Nuria Cunill ; BOZZI, Sonia Ospina. Fortalecimiento de los sistemas de monitoreo y evaluación en América Latina: informe comparativo de 12 países. Caracas: Banco Mundial/CLAD, 2008.

IAMAMOTO, Marilda. O serviço social na contemporaneidade. São Paulo: Cortez, 1998. 
IANNI, Octávio. A questão social. Revista São Paulo em Perspectiva, São Paulo, jan./mar. 1991.

JANUZZI, Paulo D. M. Monitoramento analítico como ferramenta para o aprimoramento da gestão de programas sociais. Revista Brasileira de Monitoramento e Avaliação, Brasília, DF, n. 1, jan./jun. 2011.

KAZTMAN, R.; FILGUEIRA, C. Marco conceptual sobre activos, vulnerabilidad $y$ estructura de oportunidades. Montevidéu: CEPAL, 1999. Mimeo.

KOGA, Dirce. Medidas de cidades. São Paulo: Cortez, 2003.

LANDAIS, Camille. Les hauts revenus en France (19982006): une explosion des inégalités?. Paris : Ecole d'économie de Paris, 2007. Disponible en:<http://www. altermondesansfrontiere.com//MG/pdf/rapport hauts_revenus_en_France_07_07.pdf>. Acceso en: 23 dez. $20 \overline{11}$.

LE ROY, Anne; OFFREDI, Claudine. La quantification au service de l'observation sociale locale: à quelles conditions? Revue Française de Soció́conomi, France, n. 7, 2011.

MACKAY, Keith. Como crear Sistemas de Seguimiento y Evaluación que Contribuyan a un Buen Gobierno. In: MAY, Ernesto et al. Hacia la institucionalización de los Sistemas de monitoreo y evaluación en América Latina y el Caribe. Washington, DC: Banco Mundial, 2007. Actas de la conferencia del Banco Mundial.

OFFREDI, Claudine. Itinéraire d'un questionnement de la notion d'utilité sociale en matière d'évaluation des politiques publiques. In: OFFREDI, Claudine; RAVOUX, F. (Eds.). Lanotion d'utilité sociale au défi de son identité dans l'évaluation des politiques publiques. Paris: L'Harmattan, 2010.

POCHMANN, Marcio. Nova classe média?: o trabalho na base da pirâmede social brasileira. São Paulo: Boitempo, 2012.

ROSANVALLON, Pierre. La société des égaux. Paris: Éditions du Seuil, 2011.

SILVA, Maria Ozanira de Silva e. Avaliação de políticas e programas sociais: uma reflexão sobre o conteúdo teórico e metodológico da pesquisa avaliativa. In: (Org.). Pesquisa avaliativa: aspectos teóricos metodológicos. São Paulo: Veras Editora, 2008.
SPOSATI, Aldaiza. Cidade em pedaços. São Paulo: Brasiliense, 2001.

Cidade, território, exclusão/inclusão social. [S.I.], [2000]. Disponible en:<http://www. cedest.info/marcos conceituais.html>. Acceso en: 23 dez. 2011. Texto presentado originalmente en el Congreso Internacional de Geoinformación - GEO Brasil/2000, Palácio das Convenções do Anhembi, San Pablo, 16/06/2000.

Mapa de exclusão/inclusão social da cidade de São Paulo. São Paulo: Educ,1996.

TELLES, V.; CABANES, R. (Orgs.). Nas tramas da cidade: trajetórias urbanas e seus territórios. São Paulo: Humanitas, 2006.

Igualdade: qual a medida? In: VERAS, Maura Pardini Bicudo (Org.). Hexápolis: desigualdades e rupturas sociais em metrópoles contemporâneas. São Paulo: Educ: Cortez, 2004.

VIEIRA, Evaldo. Os direitos e a política social. São Paulo: Cortez, 2009.

VIVERET, Patrick. L'enjeu démocratique dans le débat sur de nouveaux indicateurs: propos introductifs d'une audition par le Conseil Économique, Social et Environnemental. Paris: CESE, 2009. Disponible en:<http://www.idies. org/index.php?post/Lenjeu democratique dans le debat sur de nouveaux indicateurs>. Acceso en: 3 mar. 2012.

Notas

Esta reflexión es fruto del Post Doctorado financiado por CAPES, realizado en el Centro de Investigación en Economía de Grenoble (CREG) en la Universidad Pierre Mendès-France (UPMF), con orientación de la Profesora Claudine Offredi.

2 Esta crítica sobre las medidas de la pobreza será una marca apuntada por Sposati ([2000]), en diversos debates y producciones de la época.

3 ESTUDO COMPARATIVO DEL BOLSA FAMÍLIA COM OTROS PROGRAMAS DE TRANSFERENCIA DE RENTA EN AMÉRICA LATINA, realizado por la Universidad Federal de Maranhão (UFMA); la Pontificia Universidad Católica de San Pablo (PUCSP) y del Rio Grande do Sur (PUCRS), el Programa de Doctorado en Ciencias Sociales de la Facultad de Ciencias Sociales de la Universidad de la Republica/Uruguay y la Maestría en Ciencias Sociales de la Facultad de Ciencias Humanas de la Universidad Nacional del Centro de la Provincia de Buenos Aires da Argentina; coordinada por los profesores Maria Ozanira da Silva e Silva, en Brasil, Mónica de Martino Bermúdez en Uruguay e Silvia Gabriela Souto en Argentina. 
4 Para estudiar la noción de Topografia, consultar también: SPOSATI, Aldaíza. Topografia Social da Cidade de João Pessoa. 1. ed. João Pessoa: Editora Universitária/UFPB, 2010. v. 1.172 p.

5 Disponible en:<http://www.appeldesappels.org/>.

6 Disponible en:<http://www.idies.org/index.php?category/ FAIR>.

7 Al respecto, en el caso brasileño remeter a Pochman (2012).

8 En el debate francés y en la Sociedad Francesa de Evaluación esta centralidad está asociada a la noción de utilidad social, comprendida como una convención que busca recuperar la dimensión social de las políticas públicas, contraponiéndose a la dimensión mercantil que ha asumido la evaluación en las últimas décadas.

9 Los lineamientos para la implantación de la evaluación y gestión por resultados están explícitos en el estudio comparado realizado en Argentina, Bolivia, Brasil, Chile, Colombia, Costa Rica, Honduras, Méjico, Nicaragua, Paraguay, Perú y Uruguay.

\section{Carola C. Arregui}

Assistente Social

Doctora en Servicio Social por Pontificia Universidad Católica de São Paulo (PUC-SP)

E-mail: carolaarregui@gmail.com

Pontificia Universidad Católica de São Paulo (PUC-SP) Av. Sebastião Davino dos Reis, 786, Barueri -São Paulo CEP: 06414-007 